\title{
Study of intrapartum fetal distress with the help of cardiotocography and its correlation with umbilical cord blood sampling
}

\author{
S. Neeraja*, Sugathi Parimala, Naima Fathima
}

Department of Obstetrics and Gynecology, SVS Medical College, Mahabubnagar, Telangana, India

Received: 23 January 2020

Accepted: 28 February 2020

*Correspondence:

Dr. S. Neeraja,

E-mail: sreeramneeraja2904@gmail.com

Copyright: (C) the author(s), publisher and licensee Medip Academy. This is an open-access article distributed under the terms of the Creative Commons Attribution Non-Commercial License, which permits unrestricted non-commercial use, distribution, and reproduction in any medium, provided the original work is properly cited.

\begin{abstract}
Background: Even in low risk mothers, fetal acidosis occurs as in high risk groups. Aim of fetal monitoring is to detect early response to intrauterine hypoxia and prevent irreversible neurological damage and death. Objective of this study was to correlate the intrapartum fetal distress with the help of cardiotocography CTG with umbilical cord blood sampling.

Methods: A total 100 consecutive patients attending the labor ward were studied. Immediately at birth, before the baby's first breath and before delivery of the placenta, the umbilical cord blood was collected as per the standard guidelines laid down in the standard textbooks. Fetal acidosis was assessed by umbilical cord arterial blood $\mathrm{pH}$. Fetal acidosis was considered when umbilical artery $\mathrm{pH}<7.2$. Cardiotocography features were used to clinically diagnose fetal distress.

Results: Most of the mothers were multigravida. They belonged to the age group of $20-25$ years. Only $18 \%$ had abnormal CTG. Out of 50 mothers with normal vaginal delivery, all had normal CTG. Out of 43 mothers who were delivered by LSCS, no one had normal CTG, 25 had indeterminate CTG and 18 had abnormal CTG. As CTG became abnormal, proportion of mothers with the thick meconium increased. NICU admission proportion increased as CTG changed from normal to the abnormal. There was a significant association between the abnormal CTG and the umbilical cord blood $\mathrm{pH}$ being acidic.

Conclusions: CTG is a simple test, easy to perform and can alert obstetrician for necessary interventions in case of an abnormal CTG. It can detect fetal distress in labor thus helping to reduce neonatal morbidity by early intervention in cases of abnormal tracing.
\end{abstract}

Keywords: Cardiotocography, Correlation, Intrapartum fetal distress, Sampling, Umbilical cord blood

\section{INTRODUCTION}

Even in low risk mothers, fetal acidosis occurs as in high risk groups. The aim of the fetal monitoring is to detect early response to intrauterine hypoxia and prevent irreversible neurological damage and death. The acid base status of the fetus at the time of the birth can be assessed by analysis of the umbilical cord blood gas. It tells us about how the wellbeing of the fetus. There has been sharp decline in the maternal and perinatal mortality due to advanced technology being used in almost all hospitals. Intrauterine hypoxia can lead to $20-40 \%$ of the still births. They are preventable. ${ }^{1,2}$

Labor is perhaps the most stressful period that a human being endures during its lifetime. The oxygen supply to the fetus is interrupted with every uterine contraction. A healthy fetus has inbuild mechanisms to tide over these short periods of hypoxia without any consequences. However, fetus with borderline reserves due to chronic uteroplacental insufficiency may decompensate and show signs of overt asphyxia during labor. ${ }^{3,4}$ 
This study correlates the intrapartum fetal distress with the help of cardiotocography CTG with umbilical cord blood sampling.

\section{METHODS}

A total 100 consecutive patients attending labor ward in SVS Medical College and Hospital conducted from November 2016 to November 2017 at department of obstetrics and gynecology, SVS Medical College and Hospital, Mahabubnagar, Telangana, India.

\section{Inclusion criteria}

- Study subjects willing to participate

- Singleton pregnancies with vertex presentation at labor

- High risk pregnancies like anemia, hypertension, thyroid disorders, diabetes, epilepsy, asthma

- Intrauterine growth retardation, oligohydramnios, preterm deliveries.

\section{Exclusion criteria}

- Elective cesarean section

- Multiple gestation, malpresentation

- Abruptio placentae

- Intrauterine fetal death, fetus with congenital anomalies

- Maternal infections.

Institution ethics committee permission was taken. A detailed history was taken after taking the written informed consent from the study subjects and recorded in the predesigned, pretested, semi structured study questionnaire. Thorough and detailed clinical examination was carried out for all the study subjects. Electronic fetal monitor was used with a paper speed of 1 $\mathrm{cm}$ per min. fetal heart rate was recorded by external transducer and uterine contractions recorded with external tonometer applied to maternal abdominal wall.

Kocher's clamp was used to clamp the umbilical cord at two points which were approximately $10 \mathrm{~cm}$ from each other. This was done even before the baby took its first breath as soon as possible after delivery of the baby. This was also done even before the placenta was delivered. Then it was cut. 2-3 ml umbilical artery blood was collected. Fetal acidosis was assessed using the blood $\mathrm{pH}$ of blood from artery of the umbilical cord. Fetal acidosis was considered to be present if the $\mathrm{pH}$ was less than 7.2.

Cardiotocography features present in the baby were utilized to make a clinical diagnosis of distress of the fetus.

\section{Statistical analysis}

Data was analyzed by graph pad prism softer version 6.0. Descriptive data was summarized by mean+SD for continuous data and proportions for categorical data. ttest was used for continuous data and chi square for proportions. $\mathrm{p}$ value $<0.05$ was taken as statistically significant.

\section{RESULTS}

Table 1 shows distribution of study subjects as per gravida. $46 \%$ were primigravida mothers while $54 \%$ were multigravida mothers. Though the number of multigravida mothers was more than the primigravida mothers as seen from the above table in the present study but the difference was narrow and hence it can be said that the difference of numbers between the multigravida mothers and primigravida mothers is not found out to be statistically very much significant in the present study.

Table 1: Distribution of study subjects as per gravida.

\begin{tabular}{|l|ll|}
\hline Gravida & Number & Percentage \\
\hline Primigravida & 46 & $46 \%$ \\
\hline Multigravida & 54 & $54 \%$ \\
\hline Total & 100 & $100 \%$ \\
\hline
\end{tabular}

Table 2: Distribution of study subjects as per age.

\begin{tabular}{|ll|l|}
\hline Age (years) & Number & Percentage \\
\hline $18-19$ & 6 & $6 \%$ \\
\hline $20-25$ & 53 & $53 \%$ \\
\hline $26-30$ & 35 & $35 \%$ \\
\hline $31-32$ & 4 & $4 \%$ \\
\hline $33-34$ & 2 & $2 \%$ \\
\hline Total & 100 & $100 \%$ \\
\hline
\end{tabular}

Table 2 shows distribution of study subjects as per age. Majority of the mothers were in the age group of 20-25 years. This was followed by the age group of 26-30 years where 35 mothers were present. Six mothers were found to be present in the age group of 18-19 years of age. Fifty-three mothers were found to be present in the age group of 20-25 years of age. 35 mothers were found to be present in the age group of 26-30 years of age. Four mothers were found to be present in the age group of 3132 years of age. Two mothers were found to be present in the age group of 33-34 years of age.

Table 3 shows distribution of study subjects as per type of CTG (late deceleration). Only $18 \%$ of the study subjects had abnormal CTG. Fifty study subjects had normal late deceleration which is almost half of the total study subjects in the present study. Thirty-two study subjects were those in whom the late deceleration i.e. CTG could not be determined and hence they were classified as indeterminate and thus they constituted about one third of the total study subjects in the present study. Eighteen study subjects had abnormal late deceleration i.e. CTG and they constituted about $18 \%$ of the total study subjects in the present study. 
Table 3: Distribution of study subjects as per type of CTG (late deceleration).

\begin{tabular}{|lll|}
\hline Type of CTG & Number & Percentage \\
\hline Normal & 50 & $50 \%$ \\
\hline Indeterminate & 32 & $32 \%$ \\
\hline Abnormal & 18 & $18 \%$ \\
\hline Total & 100 & $100 \%$ \\
\hline
\end{tabular}

Table 4 shows relation between CTG and mode of delivery. Out of 50 study subjects with normal vaginal delivery, all 50 had normal type of CTG. Out of 43 mothers who were delivered by lower segment caesarean section delivery, no one was found to have normal type of CTG, 25 were found to have indeterminate type of CTG and 18 were found to have abnormal type of CTG. Out of 7 mothers whose babies were delivered by forceps delivery i.e. difficult delivery, no one was found to have normal type of CTG, no one was found to have abnormal type of CTG and all i.e. seven were found to have indeterminate type of CTG. Thus, if the CTG is normal, it results in normal delivery.

Table 4: Relation between CTG and mode of delivery.

\begin{tabular}{|llll|}
\hline Mode of delivery & Type of CTG & Indeterminate & Abnormal \\
\hline Normal vaginal delivery & Normal & 0 & 0 \\
\hline LSCS & 50 & 25 & 18 \\
\hline Forceps delivery & 0 & 7 & 0 \\
\hline Total & 0 & 32 & 18 \\
\hline
\end{tabular}

Table 5: CTG in relation to meconium stained liquor.

\begin{tabular}{|l|lllllll|}
\hline \multirow{2}{*}{ Normal CTG } & \multirow{2}{*}{ Number } & Clear liquor & \multicolumn{2}{c}{ Thin meconium } & \multicolumn{2}{c|}{ Thick meconium } \\
\hline Normal & 50 & 42 & $84 \%$ & 6 & $12 \%$ & 2 & $4 \%$ \\
\hline Indeterminate & 32 & 22 & $69 \%$ & 4 & $13 \%$ & 7 & $22 \%$ \\
\hline Abnormal & 18 & 7 & $39 \%$ & 3 & $17 \%$ & 8 & $44 \%$ \\
\hline
\end{tabular}

Table 6: Neonatal outcome in relation to CTG.

\begin{tabular}{|lllll|l|}
\hline CTG & Number & Apgar $<$ 7 at 5 min & Percentage & NICU admission & Percentage \\
\hline Normal & 50 & 1 & $2 \%$ & 1 & $2 \%$ \\
\hline Indeterminate & 32 & 7 & $22 \%$ & 7 & $22 \%$ \\
\hline Abnormal & 18 & 8 & $44 \%$ & 8 & $44 \%$ \\
\hline
\end{tabular}

Table 5 shows CTG in relation to meconium stained liquor. Out of 50 mothers with normal type of CTG, 42 i.e. $84 \%$ had clear liquor, six i.e. $12 \%$ had thin meconium and two i.e. $4 \%$ had thick meconium. Out of 32 mothers who had indeterminate type of CTG, 22 i.e. $69 \%$ had clear liquor, four i.e. $13 \%$ had thin meconium, seven i.e. $22 \%$ had thick meconium. Out of 18 mothers who had abnormal type of CTG, seven i.e. $39 \%$ had clear liquor, three i.e. $17 \%$ had thin meconium and eight i.e. $44 \%$ had thick meconium. Thus, it has been observed that as the CTG became abnormal, the proportion of mothers with thick meconium increased.

Table 6 shows neonatal outcome in relation to CTG. Out of 50 cases with normal CTG, only one i.e. $2 \%$ had Apgar score of less than seven at 5 min after birth and only one had neonatal intensive care unit admission. Out of 32 cases with indeterminate type of CTG, seven i.e. $22 \%$ had Apgar score of less than seven at five min after birth and seven had neonatal intensive care unit admission. Out of 18 cases with abnormal type of CTG, eight i.e. $44 \%$ had Apgar score less than seven at five min after birth and eight had neonatal intensive care unit admission. Thus, it has been observed that NICU admission proportion increased as the type of CTG changed from normal to abnormal.

Table 7: Distribution of study subjects as per umbilical cord blood $\mathrm{pH}$.

\begin{tabular}{|lll|}
\hline Umbilical cord blood pH & Number & Percentage \\
\hline Normal & 84 & $84 \%$ \\
\hline Acidosis & 16 & $16 \%$ \\
\hline Total & 100 & $100 \%$ \\
\hline
\end{tabular}

Table 7 shows distribution of study subjects as per umbilical cord blood $\mathrm{pH}$. Majority of the study subjects i.e. 84 had normal umbilical cord blood $\mathrm{pH}$. Only 16 were found to have acidotic umbilical cord blood $\mathrm{pH}$. Thus, the incidence of abnormal umbilical cord blood $\mathrm{pH}$ 
or the acidotic umbilical cord blood $\mathrm{pH}$ was found out to be only $16 \%$ in the present study.

\section{Table 8: Association between CTG and umbilical cord blood pH.}

\begin{tabular}{|lll|}
\hline CTG & Number & Acidosis $\%$ \\
\hline Normal & 50 & $2 \%$ \\
\hline Indeterminate & 32 & $22 \%$ \\
\hline Abnormal & 18 & $44 \%$ \\
\hline
\end{tabular}

Table 8 shows association between CTG and umbilical cord blood $\mathrm{pH}$. Out of 50 study subjects with normal type of CTG, only $2 \%$ had acidotic umbilical cord blood $\mathrm{pH}$. Out of 32 study subjects with indeterminate type of CTG, $22 \%$ had acidotic umbilical cord blood $\mathrm{pH}$. Out of 18 study subjects with abnormal type of CTG, 44\% had acidotic umbilical cord blood $\mathrm{pH}$.

\section{DISCUSSION}

This study found that the sensitivity $55.4 \%$, specificity $81 \%$, PPV $54.4 \%$ and NPV $45 \%$ for CTG Jackson M et al 5 found that the sensitivity $64 \%$, specificity $79 \%$, PPV $54 \%$, NPV $46 \%$ for CTG which is comparable to the findings of the present study. Visser GHA et al found that that the sensitivity $79 \%$, specificity $85 \%$, PPV $68 \%$ and NPV 91\% for CTG was which is higher than that found in the present study. ${ }^{6}$ Parveen $\mathrm{S}$ et al, found that the sensitivity, specificity, positive predictive value and negative predictive value for CTG was $15.4 \%, 86 \%$, $11.6 \%, 89 \%$ respectively which is lower than that reported in the present study.

In the present study $50 \%$ of the study subjects had category I CTG tracing which is normal, 32\% had category II tracing (intermediate), and $18 \%$ had category III i.e. abnormal CTG tracing. $89 \%$ had normal fetal baseline heart rate, $9 \%$ had bradycardia, and $2.4 \%$ had tachycardia. This was comparable to a study by Aboulghar W et al, where $82 \%$ had normal baseline fetal heart rate. ${ }^{8}$ However, the incidence of fetal bradycardia was $9 \%$ and that of tachycardia was $2.4 \%$ was different from the study by Aboulghar $\mathrm{W}$ et al, where it was reported to be much higher i.e. $17 \%{ }^{8}$

In the study by Aboulghar $\mathrm{W}$ et al the incidence of acidosis was higher with $34 \%$ of the babies having abnormal cord blood $\mathrm{pH}^{8}{ }^{8}$ Jackson $\mathrm{M}$ et al, studied the intra partum fetal heart rate characteristics of over 48,000 patients with a single ton non anomalous fetus in term labor at 10 hospitals. ${ }^{5}$ In Aboulghar W et al, where 50\% of cases with pathological CTG had acidosis and 19.2\% of cases with suspicious CTG had acidosis. ${ }^{8}$ Visser GHA et al 6 who studied normal and abnormal patterns of CTG tracing and correlated CTG findings with fetal distress after birth by umbilical cord blood gas analysis. Parveen $\mathrm{S}$ et al studied 122 cord blood samples using umbilical cord arterial base excess $(<12 \mathrm{mmol} / \mathrm{l})$ at birth to diagnose fetal acidemia. ${ }^{7}$
In the present study $44.4 \%$ had abnormal beat to beat variability, (35.8\% of the cases had minimal variability, $12.4 \%$ had absent variability, and $4 \%$ had increased variability). This value of abnormal fetal heart rate variability on CTG is slightly less than that in a study by Aboulghar W et al, where it was $55 \%{ }^{8}$

In the present study, accelerations in CTG were present in $52.6 \%$ of cases where in study by Aboulghar W et al, it was found to be only $6 \%{ }^{8}$ Decelerations in CTG were present in $17.9 \%$ of cases which is comparable to study by Modarressnejad V et al, it was $14.3 \%$. ${ }^{9}$ It was higher in study by Aboulghar $\mathrm{W}$ et al, i.e. $53 \%{ }^{8}$

In the present study mean cord blood $\mathrm{pH}$ was $7.2 \pm 0.07$ which was comparable to other studies. $16 \%$ of neonates had acidosis which is comparable to studies by Kaban A et al (13.26\%) and Modarressnejad V et al it was $20.3 \%$, Aboulghar W et al, it was $34 \%$ which is higher. ${ }^{8-10}$

Jackson $\mathrm{M}$ et al, found that intrapartum fetal heart rate was category I in $77.9 \%$, category II in $22.1 \%$, and category III in $0.004 \%$ of time. ${ }^{5}$ In 2 hours before delivery, category I tracing were less commonly observed $(60.9 \%)$, and both category II and III tracings became more common. They concluded that category I and II fetal heart rate patterns are more common in labor than in category III. These results are comparable to the findings of the present study.

In the present study, $44 \%$ of the cases with category III (abnormal) CTG had acidosis and 22\% cases with category II (intermediate) CTG had acidosis. A significant association was found between type of CTG and cord blood $\mathrm{pH}$. Aboulghar $\mathrm{W}$ et al, noted similar observations. $^{8}$

In the present study it was found that abnormal fetal heart rate had 3.1 times higher risk for abnormal cord blood $\mathrm{pH}$ and bradycardia had 2.8 times higher risk for abnormal cord blood $\mathrm{pH}$. These findings are comparable with those by Aboulghar $\mathrm{W}$ et al. ${ }^{8}$

An abnormal CTG had sensitivity of $46.5 \%$, specificity $91.6 \%$, in detecting acidosis which meant that while an abnormal CTG tracing could detect $46 \%$ of subjects with acidosis, it had a good ability to identify those who did not have acidosis because of its high specificity. It was noted that an abnormal CTG had PPV $=62.5 \%$ and NPV $=85.4 \%$ which meant that in the absence of abnormal CTG, the chance of having acidosis was very minimal. Parveen $\mathrm{S}$ et al, noted similar observations. ${ }^{7}$

Visser GHA et al, found sensitivity $79 \%$, specificity $85 \%$, PPV $68 \%$, NPV $91 \%$ for CTG. ${ }^{6}$ Steer PJ et al conducted a prospective study to find a correlation among FHR patterns, MSL, umbilical cord arterial blood $\mathrm{pH}$, and Apgar score in 698 cases and found that indeterminate CTG had sensitivity $22.7 \%$, specificity $92.7 \%$ which meant that indeterminate CTG could diagnose only 
$22.7 \%$ of cases with acidosis. ${ }^{11}$ However, from its high specificity, it also had a good ability to identify those who did not have acidosis. ${ }^{11}$

However, when abnormal and indeterminate CTG were taken together, they were found to have $80 \%$ sensitivity in detecting acidosis with $56.39 \%$ specificity, $29.3 \%$ PPV, and $92.7 \%$ NPV. It means that in the absence of abnormal and indeterminate CTG there was less chance of having fetus without acidosis. Present study also found that a normal CTG had 55.4\% sensitivity, $81 \%$ specificity, 90.4\% PPV, 25.8\% NPV, for diagnosing normal cord blood $\mathrm{pH}$. This meant that a normal CTG can accurately detect babies without acidosis. These findings are comparable to those found by Parveen $\mathrm{S}$ et al, who concluded that a normal CTG trace correlates highly with absence of fetal acidosis. ${ }^{7}$ They found that cardiotocography had $15.38 \%$ sensitivity, $86 \%$ specificity, 11.6\% PPV, 89\% NPV.

Thus, from the findings of the present study although sensitivity of CTG was low, high specificity, low cost, and ease of carrying out monitoring supported in altering the obstetrician regarding an intra uterine hypoxic event.

\section{CONCLUSION}

CTG is a simple test, easy to perform and can alert obstetrician for necessary interventions in case of an abnormal CTG. It can detect fetal distress in labor thus helping to reduce neonatal morbidity by early intervention in cases of abnormal tracing. Thus, from the findings of the present study although sensitivity of CTG was low, high specificity, low cost, and ease of carrying out monitoring supported in altering the obstetrician regarding an intra uterine hypoxic event.

Funding: No funding sources Conflict of interest: None declared

Ethical approval: The study was approved by the Institutional Ethics Committee

\section{REFERENCES}

1. McLennan A. A template for defining a causal relationship between acute intrapartum events and cerebral palsy. In: Bonnar J, editor. Recent Advances in Obstetrics and Gynecology. Churchill Livingstone; 2001;21:86-93.
2. Macones G, Hankins G, Spong C, Hauth J, Moore T. The 2008 national institute of child health and human development workshop report on electronic fetal monitoring. Update on definitions, interpretations and guidelines. Obstet Gynecol. 2008;112(3):661.

3. Macones GA. Intrapartum fetal heart rate monitoring: nomenclature, interpretation and general management principles. Obstet Gynecol. 2009; 114(1):192-202.

4. Yeh P, Emary K, Empray L. The relationship between umbilical cord arterial $\mathrm{pH}$ and serious adverse neonatal outcome: analysis of 51519 consecutive validated samples. Br J Obstet Gynecol. 2012;119:824-31.

5. Jackson M, Holengrem CM, Espen MS, Henry E, Varner MW. Frequency of fetal heart rate categories and short-term neonatal outcome. Obstet Gynecol. 2011;118(4):803-8.

6. Visser GHA, Sadovsky G, Nicolaids KH. Antepartum fetal heart rate patterns in small fetus gestational age third obtained at cordocentesis. Am J Obstet Gynecol. 1990;162(3):696-703.

7. Parveen S. Umbilical cord arterial blood base excess as gold standard for fetal well-being screening test at term delivery. J Pak Med Assoc. 2010;60(5):347-50.

8. Aboulghar WM, Ibrahim MA, Allam IS, Hosny W, Otify M. Validity of cardiotocography in the diagnosis of acute fetal hypoxia in low resource settings. Internet J Gynecol Obstet. 2013;17(1):1-8.

9. Modarressnejad V. Umbilical cord blood $\mathrm{pH}$ and risk factors for acidemia in neonates. Eastern Mediterranean Health J. 2005;11:96-101.

10. Kaban A, Cengiz H, Kaban I, Ozcan A, Karakas S. The success of cardiotocography in predicting perinatal outcome. $\mathrm{J}$ Clin Experiment Invest. 2012;3(2):168-71.

11. Steer PJ, Eigbe F, Lissauer TJ, Beard RW. Inter relationships among abnormal cardiotocograms in labor, meconium staining of the amniotic fluid, arterial cord blood $\mathrm{pH}$ and APGAR scores. Obstet Gynecol. 1989;74(5):715-21.

Cite this article as: Neeraja $\mathrm{S}$, Parimala $\mathrm{S}$, Fathima N. Study of intrapartum fetal distress with the help of cardiotocography and its correlation with umbilical cord blood sampling. Int J Reprod Contracept Obstet Gynecol 2020;9:1580-4. 